\title{
Comparison of SPIHT and Lifting Scheme Image Compression Techniques for Satellite Imageries
}

\author{
Nagamani.K \\ Assistant Professor, \\ $\mathrm{R} V$ College of Engineering, \\ Bangalore-56005.
}

\author{
A.G Ananth \\ Professor, \\ $\mathrm{R} V$ College of Engineering, \\ Bangalore-560059.
}

\begin{abstract}
Wavelets offer an elegant technique for representing the levels of details present in an image. When an image is decomposed using wavelets, the low pass components carry more information than the high pass components. The possibility of better elimination of high pass components gives higher compression ratio for wavelet based techniques. To achieve higher compression ratio, various coding schemes have been considered. The study of 2-D Discrete Wavelet Technique (DWT) architectures reveals that there are two schemes for implementing DWT, one is based on convolution and other based on lifting scheme. . In the present paper a detailed study of the lifting compression scheme for satellite imageries has been carried out. A comparison between the performance of the lifting scheme and SPHIT technique has been made in the context of satellite imageries. For a given compression ratio, the PSNR (peak signal to noise ratio) values are estimated for both the schemes to achieve better quality of the reconstructed image. The results of the analysis demonstrate that for both the schemes, the PSNR values increases with the level of decomposition. The results of the analysis further indicates that for satellite imageries, the lifting scheme is more efficient for obtaining higher compression ratio $\sim 8$ and better PSNR values $\sim 29$ for achieving good quality of the reconstructed image.
\end{abstract}

Keywords: DWT, PSNR, EZW, SPIHT, LIFTING SCHEME,

\section{INTRODUCTION}

The SPIHT algorithm, developed by Said and Pearlman in 1996 [2] is a fast and efficient image compression algorithm works by testing ordered wavelet coefficients for significance in a decreasing bit plane order, and quantizing only the significant coefficients. The high coding efficiency obtained by this algorithm is due to a group testing of the coefficients of a wavelet tree. The SPIHT (Set Partitioning in Hierarchical Trees) algorithm is a refined version of EZW algorithm. It can perform better at higher compression ratios for a wide variety of images than EZW. The algorithm uses a partitioning of the trees in a manner that tends to keep insignificant coefficients together in larger subsets.

The SPIHT algorithm groups the wavelet coefficients and trees into sets based on their significance information. The encoding algorithm consists of two main stages, sorting and refinement. In the sorting stage, the threshold for significance is set as $2 \mathrm{n}$, where $\mathrm{n}$ is the bit level, and its initial value is determined by the number of bits required to represent the wavelet coefficient with the maximum absolute value. Significance for trees is obtained by checking all the member detail coefficients. Different architectures have been studied and performance parameters such as PSNR and Compression Ratio are determined. After obtaining double precision value of the image of size $256 * 256$ imagery in BMP format, discrete wavelet transforms techniques are applied to obtain the wavelet coefficients for calculating PSNR and Compression ratio. Inverse Discrete wavelet transform are applied to get back the reconstructed image $[10,11,2,13,14]$

\section{SPIHT ALGORITHM}

The data structure used by the SPIHT algorithm is similar to that used by the EZW algorithm. The wavelet coefficients are again divided into trees originating from the lowest resolution band (band I). The coefficients are grouped into $2 \times 2$ arrays that, except for the coefficients in band I, are off springs of a coefficient of a lower resolution band. The coefficients in the lowest resolution band are also divided into $2 \times 2$ arrays. However, unlike the EZW case, all but one of them are root nodes. The coefficient in the top-left comer of the array does not have any offspring.

The trees are further partitioned into four types of sets, which are sets of coordinates of the coefficients:

O (i, j) This is the set of coordinates of the offsprings of the wavelet coefficient at location ( $i, j)$.

$\mathrm{D}(\mathrm{i}, \mathrm{j})$ This is the set of all descendants of the coefficient at location $(\mathrm{i}, \mathrm{j})$

$\mathrm{H}$ is set of all root nodes.

$\mathrm{L}(\mathrm{i}, \mathrm{j})$ This is the set of coordinates of all the descendants of the coefficient at location except for the immediate offsprings of the coefficient at location $(i, j)$. So,

$$
L(i, j)=D(i, j)-Q(i, j)
$$

A set D (i, j) or $L(i, j)$ is said to be significant if any coefficient in the set has a magnitude greater than the threshold. The algorithm makes use of three lists: the list of insignificant pixels (LIP), the list of significant pixels (LSP), and the list of insignificant sets (LIS). The LSP and LIS lists contain the coordinates of coefficients, while the LIS contains the coordinates of the roots of sets of type $D$ or $L$. The initial value of the threshold is given as

$$
n=\log _{2} C_{\max }
$$


Where, $\mathrm{C}_{\max }$ is the maximum magnitude of the coefficients to be encoded. The LIP list is initialized with the set $H$. Those elements of $H$ that have descendants are also placed in LIS as type $D$ entries. The LSP list is initially empty. each pass, the members of the LIP are first processed, then the members of LIS. This is essentially the significance map encoding step. In the refinement step the elements of LSP are processed. Each coordinate contained in LIP is examined first. If the coefficient at that coordinate is significant (i.e., it is greater than $2^{\mathrm{n}}$ ), a 1 is transmitted, followed by a bit representing the sign of the coefficient ( 1 for positive, 0 for negative). Then that coefficient is moved to the LSP list. If the coefficient at that coordinate is not significant, a 0 is transmitted. After examining each coordinate in LIP, the sets in LIS are examined. If the set at coordinate $(\mathrm{i}, \mathrm{j})$ is not significant, a 0 is transmitted. If the set is significant, a 1 is transmitted. If the set is of type $D$, each of the offsprings of the coefficient at that coordinate is checked.

For each coefficient that is significant, a 1 is transmitted, the sign of the coefficient, and then the coefficient is moved to the LSP. For the rest a 0 is transmitted and their coordinates are added to the LIP. If this set is not empty, it is moved to the end of the LIS and marked as type $D$. This new entry into the LIS has to be examined during this pass. If the set is empty, the coordinate $(\mathrm{i}, \mathrm{j}$ ) is removed from the list. If the set is of type $D$, each coordinate in $D(i, j)$ is added to the end of the LIS as the root of a set of type $D$. Again, note that these new entries in the LIS have to be examined during this pass. Then $(i, j)$ is removed from the LIS. Once each of the sets in the LIS (including the newly formed ones) is processed, a refinement step is started. In the refinement step each coefficient that was in the LSP prior to the current pass is examined and output the nth most significant bit of $\left|c_{i, j}\right|$.

The coefficients that have been added to the list in this pass are ignored because, by declaring them significant at this particular level, the decoder has already been informed of the value of the nth most significant bit. This completes one pass. Depending on the availability of more bits or external factors, $n$ is decremented by one and the process continues.

\section{LIFTING SCHEME}

The lifting scheme formally introduced by Sweldens ${ }^{1}$ is a wellknown method to create bi-orthogonal wavelet filters from other ones. Usually, a poly-phase decomposition (or lazy wavelet transform, LWT) of the input signal $\mathrm{x} 0$ into sub signals is done, obtaining an approximation signal $\mathrm{x}$ and a detail signal $\mathrm{y}$. Then, lifting steps are performed by predicting the detail signal from the $\mathrm{x}$ samples and updating the approximation signal with the $\mathrm{y}$ samples .The so-called prediction P (or dual lifting) and update $U$ (or primal lifting) steps improve the initial lazy wavelet properties.[1,2,3,4,5,6,7,8,9]

$$
\begin{aligned}
& y_{0}[n]=y[n]-p(x[n]) \\
& x_{0}[n]=x[n]+U\left(y_{0}[n]\right)
\end{aligned}
$$

Although every reconstruct able filter bank can be expressed in terms of lifting steps, an explicit decomposition for a family of wavelets is only known for the Cohen-Daubechies-Feauveau wavelet.
Peak Signal to Noise Ratio (PSNR): The PSNR is calculated with the following formula

$$
P S N R=10 \log _{10}\left[\frac{\text { MaxgreyLere }{ }^{*} M N}{\sum_{x y}|g(x, y)-f(x, y)|}\right]
$$

Where $\mathrm{g}(\mathrm{x}, \mathrm{y})$ is the compressed image, $\mathrm{f}(\mathrm{x}, \mathrm{y})$ is the raw image , $\mathrm{M}$ is the image width, $\mathrm{N}$ is the image height and max. gray level is the max. value of $\mathrm{f}(\mathrm{x}, \mathrm{y})$. A Max grey level $=255$ has been used (as there are 0 to 255 grey levels represented with 8 bits in the BMP format images).

\section{RESULTS AND DISCUSSION}

The lifting algorithm was implemented by writing Matlab codes to analyze and synthesize the image in BMP format. For a given image, the lifting algorithm performs the forward DWT to get the filter coefficients ( analysis step) and encodes the image. The inverse DWT is performed with the same lifting algorithm to reconstruct the original image (synthesis step). The lifting algorithm is applied to three test images of $256^{*} 256$ grey scale imageries i.e. 'lena. bmp', 'satellite rural..bmp' and 'satellite urban..bmp'. The three test images include the standard Lena image, Satellite urban and Satellite rural imageries have been used for comparison of the two compression techniques namely SPIHT and Lifting schemes.

Using the lifting scheme algorithms, the DWT coefficients are derived for the three images. For compression ratio of $\sim 8$, the PSNR values are computed for different levels of decomposition. The results are as shown in the Figure 2 as well as in the Table 1, $2 \& 3$.

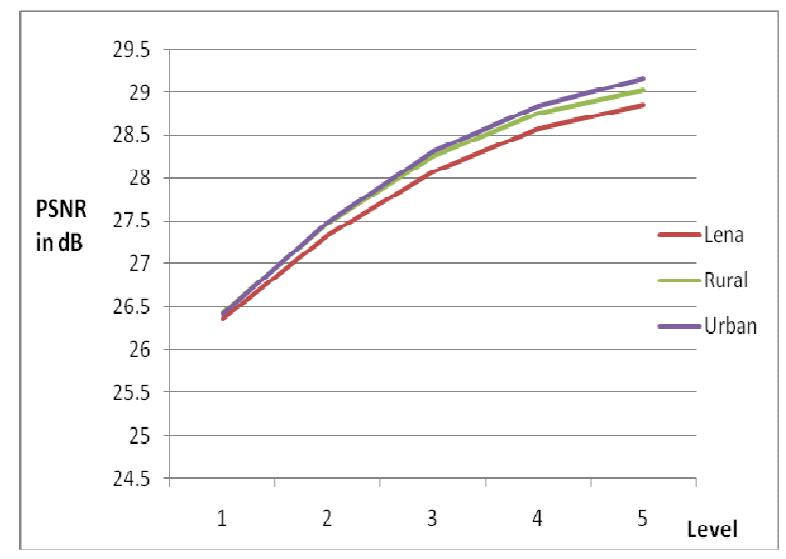

Figure 2. Plot of PSNR in $\mathrm{db} \mathrm{v} / \mathrm{s}$ level for the three different images under study using Lifting scheme. 
Table 1. The variation of PSNR in $\mathrm{dB}$ and compression ratio for the LENA image using Lifting scheme

\begin{tabular}{|c|c|c|c|}
\hline $\begin{array}{c}\text { Image } \\
\text { Type }\end{array}$ & Level & $\begin{array}{c}\text { PSNR in } \\
\text { dB }\end{array}$ & $\begin{array}{c}\text { Compression } \\
\text { ratio }\end{array}$ \\
\hline Lena & 1 & 26.3672 & 8 \\
\cline { 2 - 4 } & 2 & 27.3388 & 8 \\
\cline { 2 - 4 } & 3 & 28.0779 & 8 \\
\cline { 2 - 4 } & 4 & 28.5664 & 8 \\
\cline { 2 - 4 } & $\mathbf{5}$ & 28.8505 & 8 \\
\hline
\end{tabular}

Table 2. The variation of PSNR in $\mathrm{dB}$ and compression ratio for the satellite urban image using Lifting scheme

\begin{tabular}{|l|c|c|c|}
\hline Image & Level & PSNR in dB & Compression ratio \\
\hline Urban & 1 & 26.4170 & 8 \\
\cline { 2 - 4 } & 2 & 27.4908 & 8 \\
\cline { 2 - 4 } & 3 & 28.3176 & 8 \\
\cline { 2 - 4 } & 4 & 28.8490 & 8 \\
\cline { 2 - 4 } & 5 & 29.1629 & 8 \\
\hline
\end{tabular}

Table 3. The variation of PSNR in $\mathrm{dB}$ and compression ratio for the satellite rural image using Lifting scheme

\begin{tabular}{|c|c|c|c|}
\hline Image & Level & PSNR in dB & $\begin{array}{c}\text { Compression } \\
\text { ratio }\end{array}$ \\
\hline Rural & 1 & 26.4255 & 8 \\
\cline { 2 - 4 } & 2 & 27.4734 & 8 \\
\cline { 2 - 4 } & 3 & 28.2423 & 8 \\
\cline { 2 - 4 } & 4 & 28.7420 & 8 \\
\cline { 2 - 4 } & 5 & 29.0126 & 8 \\
\hline
\end{tabular}

It is observed from the Figure 2 and Table 1, 2 and 3 that, for the compression ratio, $\sim 8$, the PSNR values computed for all the images show slow variation with increase of decomposition levels form 1-5. The range of PSNR values variation observed are with in $10 \%$ of the maximum value. The maximum PSNR values achieved for the three types of images are $\sim 29$. For the Satellite rural and urban imageries the saturation of PSNR values occurs by the $3^{\text {rd }}$ level. This indicates that for lifting scheme, by increasing the level of decomposition one cannot achieve higher PSNR values for satellite imageries. The compression ratio $\sim 8$ remains constant for both satellite urban and rural images and the PSNR values are high and produce very good quality of the images irrespective of the origin of the image production. The lifting scheme is ideally suited for compression of satellite imageries for achieving high compression ratio and good quality for the reconstructed images.

\section{COMPARISON BETWEEN LIFTING AND SPIHT ALGORITHM}

The SPIHT algorithm has been implemented on MATLAB software and tested for three sample images namely: standard Lena image, satellite rural image, satellite urban image. The bit rate is kept constant and level of decomposition is varied from level 1 to level 5. For a maximum compression ratio $\sim 8$ and for different levels of decomposition the PSNR values are determined for the three types of images.
The PSNR values derived for different levels of decomposition using the SPIHT algorithm and Lifting scheme for the Lena Image, satellite urban and satellite rural images are shown in Table 4, 5 and 6. For comparison purpose graphs are plotted for the variation of PSNR values with different levels of decomposition derived from both SPIHT and Lifting schemes are shown in Figures 4, 5 and 6.

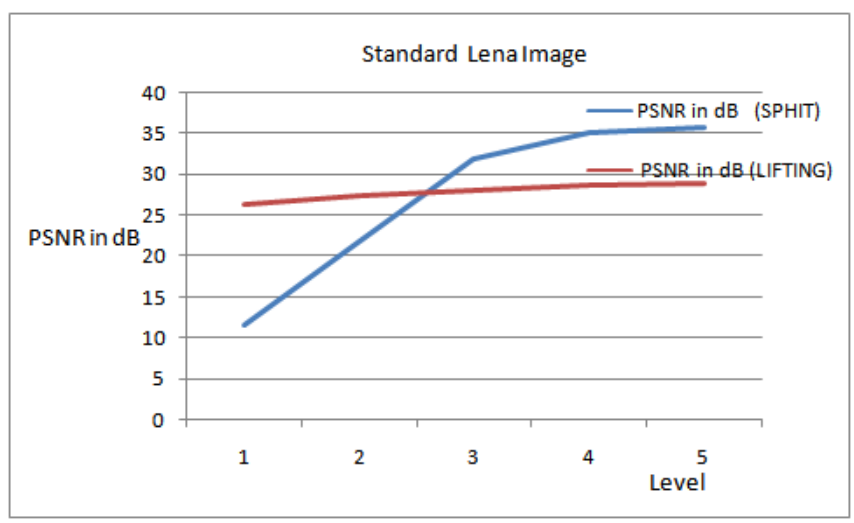

Figure 4. Shows the PSNR variation with decomposition levels derived for SPIHT and Lifting Scheme for Lena image

It is evident from the table 4 and Figure 4 that for the Lena Image the PSNR values derived from SPIHT algorithm increases rapidly from 11.60 to $35.81 \mathrm{~dB}$ for decomposition levels from 1-5 and shows a peak value of $\sim 35.81 \mathrm{~dB}(32 \%)$ for the $5^{\text {th }}$ level of decomposition. Where as for the lifting scheme the PSNR values shows a gradual increase in PSNR values from 26.36 and reaches a steady value of $28.08 \mathrm{~dB}$ at the $3{ }^{\text {rd }}$ level of decomposition and peak value of $228.85 \mathrm{~dB}(97 \%)$ for the $5^{\text {th }}$ level of decomposition .

Table 4. The comparison of variation of PSNR in $\mathrm{dB}$ for SPHIT and LIFTING schemes for the standard LENA image (Compression Ratio 8)

\begin{tabular}{|l|c|c|c|}
\hline $\begin{array}{l}\text { Image } \\
\text { Type }\end{array}$ & Level & $\begin{array}{l}\text { PSNR in dB } \\
\text { (SPHIT) }\end{array}$ & $\begin{array}{l}\text { PSNR in dB } \\
\text { (LIFTING) }\end{array}$ \\
\hline \multirow{4}{*}{ Lena } & 1 & 11.60 & 26.3672 \\
\cline { 2 - 4 } & 2 & 21.96 & 27.3388 \\
\cline { 2 - 4 } & 3 & 31.95 & 28.0779 \\
\cline { 2 - 4 } & 4 & 35.25 & 28.5664 \\
\cline { 2 - 4 } & 5 & 35.81 & 28.8505 \\
\hline
\end{tabular}

Similarly for satellite urban image using the SPIHT and Lifting scheme algorithm, the PSNR values has been obtained for different decomposition levels. The PSNR values derived for the image are tabulated in Table 5. The Figure 5 shows the comparison of the two PSNR values for satellite urban image. It is very evident from the table and Figure that the PSNR values derived form SPIHT techniques rapidly increases form $5.7 \mathrm{~dB}$ to $19.0 \mathrm{~dB}$ with the increase of decomposition levels from 1-5.and attains a maximum PSNR value $\sim 19.0 \mathrm{~dB}(30 \%)$. Where as the PSNR value for lifting algorithms shows a gradual increase from 26.41 with level of decomposition and indicates steady PSNR value $\sim 28.31 \mathrm{~dB}$ for the $3^{\text {rd }}$ level of decomposition and a peak 
value $\sim 29.16 \mathrm{~dB}$ ( $97 \%$ ) for the $5^{\text {th }}$ level of decomposition. In both the cases further increase in the level of decomposition of the image will not show any improvement in the PSNR.

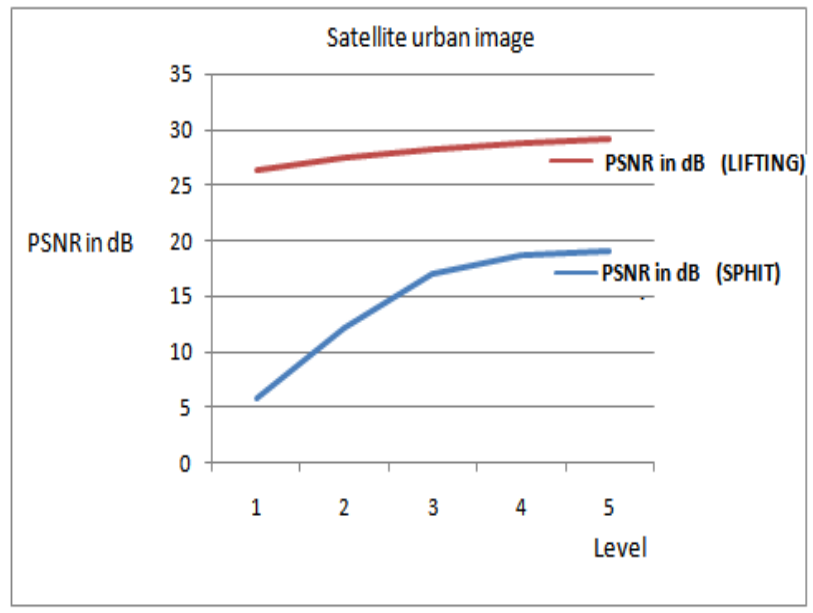

Figure 5. Shows the PSNR variation with decomposition levels derived for SPIHT and Lifting Scheme for Satellite urban image

The PSNR value derived for a satellite rural image at different decomposition levels using the SPIHT algorithm along with the PSNR values derived from Lifting scheme for the same image are shown in the Table 6. The comparison of the PSNR values derived form the two techniques are shown in Figure 6. It is clearly evident from the Table and Figure that the PSNR values derived from SPIHT techniques rapidly increases form 3.7 to 12.6 $\mathrm{dB}$ with the increase of decomposition levels from 1-5.and attains a peak PSNR value of $\sim 12.6 \mathrm{~dB}(29 \%)$ for the $5^{\text {th }}$ decomposition level. Where as the PSNR derived for lifting algorithms shows a gradual increase in PSNR value from 26.42 and reaches a steady PSNR value $\sim 28.24 \mathrm{~dB}$ at the $3^{\text {rd }}$ level of decomposition and peak value $\sim 29.01 \quad$ (97\%). Further increase in the level of decomposition of the image will not show any improvement in the PSNR.

It is evident from the Table 5, 6 that for the SPIHT scheme the maximum PSNR values achievable for both satellite urban and rural image is only $\sim 13 \mathrm{~dB}$. In this case the image quality of the reconstructed image will not be very good and cannot be improved further even with the increase of levels of decomposition. Where as the same Table 5, 6 show that for the Lifting scheme for both satellite urban and rural images one can achieve higher values of the PSNR $\sim 28 \mathrm{~dB}$ even with $3^{\text {rd }}$ level of decomposition.

These results conclusively demonstrate that the lifting schemes achieves higher PSNR values and better quality of the both urban and rural reconstructed satellite imageries compared to that of SPIHT algorithms.
Table 5. The comparison of variation of PSNR in $\mathrm{dB}$ for SPHIT and LIFTING schemes for Satellite Urban image (Compression Ratio 8)

\begin{tabular}{|c|c|c|c|}
\hline $\begin{array}{l}\text { Image } \\
\text { Type }\end{array}$ & Level & $\begin{array}{c}\text { PSNR in dB } \\
\text { (SPHIT) }\end{array}$ & $\begin{array}{c}\text { PSNR in dB } \\
\text { (LIFTING) }\end{array}$ \\
\hline \multirow{4}{*}{ Urban } & 1 & 5.70 & $\mathbf{2 6 . 4 1 7 0}$ \\
\cline { 2 - 4 } & 2 & 12.06 & $\mathbf{2 7 . 4 9 0 8}$ \\
\cline { 2 - 4 } & 3 & 17.00 & $\mathbf{2 8 . 3 1 7 6}$ \\
\cline { 2 - 4 } & 4 & 18.55 & $\mathbf{2 8 . 8 4 9 0}$ \\
\cline { 2 - 4 } & 5 & 19.00 & $\mathbf{2 9 . 1 6 2 9}$ \\
\hline
\end{tabular}

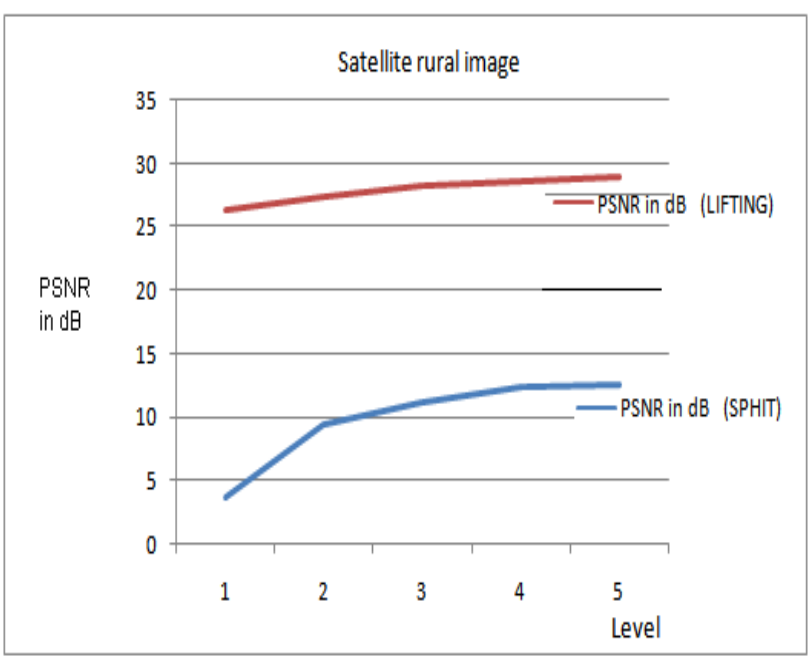

Figure 6. Shows the PSNR variation with decomposition levels derived for SPIHT and Lifting Scheme for Satellite rural image

Table 6. The comparison of variation of PSNR in $\mathrm{dB}$ for SPHIT and LIFTING schemes for Satellite Rural image (Compression Ratio 8)

\begin{tabular}{|c|c|c|c|}
\hline $\begin{array}{l}\text { Image } \\
\text { Type }\end{array}$ & Level & $\begin{array}{c}\text { PSNR in dB } \\
\text { (SPHIT) }\end{array}$ & $\begin{array}{c}\text { PSNR in dB } \\
\text { (LIFTING) }\end{array}$ \\
\hline \multirow{4}{*}{ Rural } & 1 & 3.70 & $\mathbf{2 6 . 4 2 5 5}$ \\
\cline { 2 - 4 } & 2 & 9.47 & $\mathbf{2 7 . 4 7 3 4}$ \\
\cline { 2 - 4 } & 3 & 11.25 & $\mathbf{2 8 . 2 4 2 3}$ \\
\cline { 2 - 4 } & 4 & 12.40 & $\mathbf{2 8 . 7 4 2 0}$ \\
\cline { 2 - 4 } & 5 & 12.60 & $\mathbf{2 9 . 0 1 2 6}$ \\
\hline
\end{tabular}




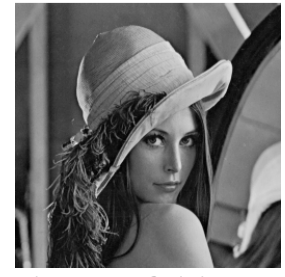

Figure 7 a Original LENA

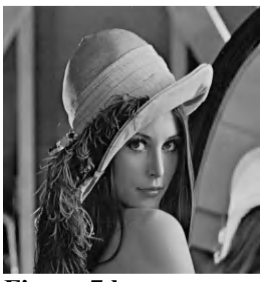

Reconstructed for level 5(SPIHT

ALGORITHM) PSNR = 35.81

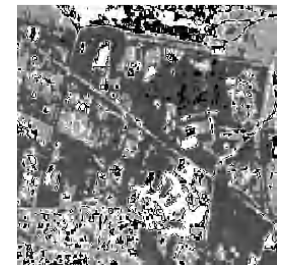

Figure 8 b

Reconstructed for

level 5(SPIHT

ALGORITHM)

PSNR $=19 \mathrm{db}$
Figure 7 b

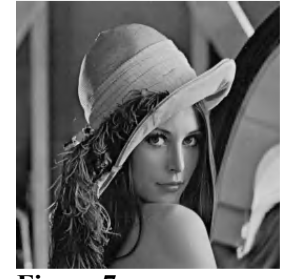

Figure 7 c

Reconstructed for level 5(LIFTING SCHEME ) $P S N R=28.8 \mathrm{db}$

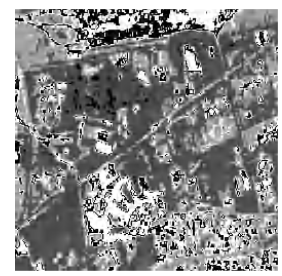

Figure 8 c

Reconstructed for level 5(LIFTING SCHEME ) $\mathrm{PSNR}=29.16 \mathrm{~dB}$
The original Lena image and the reconstructed Lena images using the two different compression schemes namely SPIHT algorithm and Lifting scheme for compression ratio of $\sim 8$ is shown in Figure 7. It is clearly evident from the Figure that reconstructed Lena image shows better quality of the image for SPIHT scheme (Figure 7b ) compared to that of Lifting scheme ( Figure (Fig 7c ) as the PSNR values achievable for SPIHT scheme is much higher than that of Lifting scheme. For standard images the Lifting schemes do not show better performance compared to the SPIHT. The results indicate that In the case of standard Lena image one can achieve high compression ratio and high PSNR values and better image quality for both SPIHT and lifting scheme compression technique.

Similarly for the original satellite urban image the reconstructed urban images using the SPIHT algorithm and lifting scheme for compression ratio $\sim 8$ is shown in Figure 8 . It is clearly seen from the Figure $8 \mathrm{c}$ that the reconstructed image for lifting scheme shows better quality of the urban image compared to that of SPIHT image shown in Figure $8 \mathrm{~b}$ as the PSNR values determined are much higher for the Lifting scheme $(\sim 29.16 \mathrm{~dB})$. For satellite urban image the Lifting schemes show better performance compared to the SPIHT. For satellite urban images

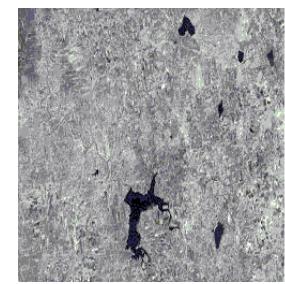

Figure 9 a Original Image

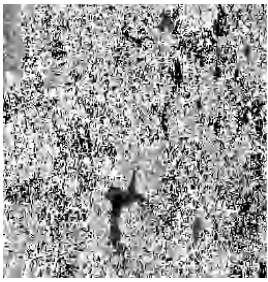

Figure 9 b level 5(SPIHT ALGORITHM) PSNR $=12.6 \mathrm{~dB}$ Reconstructed for

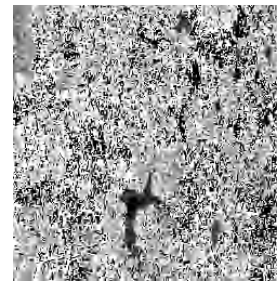

Figure 9 c Reconstructed for level 5(LIFTING SCHEME ) PSNR $=29.0126 \mathrm{~dB}$ one can achieve high compression ratio, PSNR values and better image quality using lifting scheme.

The original satellite rural image and the reconstructed rural images after compression using the SPIHT algorithm and lifting scheme for compression ratio $\sim 8$ is shown in Figure 9. The figure demonstrates that the reconstructed image for lifting scheme shows better quality of the rural image compared to that of SPIHT image as the PSNR values are higher for the Lifting scheme $(\sim 29.01 \mathrm{~dB})$, It is found that even for satellite rural images the lifting scheme gives better compression ratios, higher PSNR values and better quality of the reconstructed image.

It is evident from the Figures 7, 8 and 9 that the Lifting scheme the is most efficient technique for compression of satellite images for both rural and urban imageries the PSNR values achieved for these images are very high and the quality of the reconstruction of the images are much better than those obtained using SPIHT algorithms. Further the results of the present work illustrates that for satellite imageries high PSNR values are achieved for Lifting schemes at much lower decomposition levels

\section{CONCLUSIONS}

From the results and discussion presented the following conclusions can be drawn.

1. Lifting schemes are better suited and can be easily adopted for the compression of both satellite urban and rural imageries for achieving significant compression ratios.

2. The PSNR values derived from the SPIHT algorithms for both satellite urban and rural imageries indicate considerably lower values $\sim 19 \mathrm{~dB}$ even at higher decomposition levels 5, suggesting that using the SPIHT, it is difficult to achieve higher quality of the reconstructed image.

3. Using the lifting schemes for satellite urban and rural imageries one can achieve higher PSNR values $\sim 29 \mathrm{~dB}$ even for lower decomposition level 3 , indicating that the lifting schemes are better suited for the compression of satellite imageries.

4. The lifting scheme can produce higher compression ratios $(\sim 8)$, higher PSNR values $(\sim 29 \mathrm{~dB})$ and better quality for the reconstructed satellite imageries even at lower levels of decomposition.

\section{ACKNOWLEDGEMENT}

The Authors are thankful to Dr Ganesh Raj, Dy Director, ISRO for providing the satellite images and Head, Dept of Telecommunication, RV Engineering college for encouragement in carrying out the present research work.

\section{REFERENCE}

[1] Swelden, “ A Fast Progressive Image Sampling UsingLifting Scheme And Non-Uniform B-Splines", ISIE 2007. IEEE International Symposium on Industrial Electronics. 4-7 June 2007. 
[2] Wavelets and Filter Banks by Gilbert Strang and Truong Nguyen, Wellesley-Cambridge Press, 1997

[3] Ripples in Mathematics: the Discrete Wavelet Transform by Arne Jense and Anders la Cour-Harbo, Springer, 2001

[4] 4. Colm Mulcahy, "Image Compression Using The Haar Wavelet Transform" , Spelman College Science \& Mathematics Journal, Vol 1, No 1, April 1997, 22-31.

[5] M L Hilton, B D Jawerth and Ayan Sengupta, "Compressing still and moving images with wavelets" published in the journal "Multimedia Systems" vol-2, no-3, 1994.

[6] P J Burt and E H Adelson "The Laplacian Pyramid as a compact image code" IEEE Trans. on Communication. Vol 31, pp 532-540, (1983)

[7] Bryan Usevitch, "A Tutorial on Modern Lossy Wavelet Image Compression : Foundations of JPEG 2000," IEEE Signal Processing Magazine, 2001.

[8] R. A. DeVore, B. Jawerth, and B. J. Lucier, "Image Compression Through Wavelet Transform Coding," IEEE Trans. On Information Theory, Vol. 38, No. 2, pp. 719 - 746, (1992).

[9] A. S. Lewis and G. Knowles, "Image Compression Using the 2-D Wavelet Transform", IEEE Trans. on Image Processing, Vol. 1, No. 2, pp. 244-250, April (1992).

[10] J.M.Shapiro, “An Embedded Wavelet Hierarchical Image Coder," Proc. IEEE Int. Conf. Acoust. Speech, Signal Processing, San Francisco, CA, (1992).

[11] J.M.Shapiro,"Embedded Image Coding Using Zerotrees of Wavelet Coefficients", IEEE Trans. on Signal Processing, Vol. 41, pp 3445-3462, (1993)

[12] 12. M Vetterli, J Kovacevic and D J Legall “Perfect Reconstruction Filter Banks for HD TV representation and coding" Image communication Vol 2 essing Magazine . Vol 8, pp 349-364 (1990)

[13] 13. A. Said and W.A. Pearlman, "A New, Fast and Efficient Image Codec Based on Set Partitioning in Hierarchical Trees", IEEE Trans. on Circ and Syst for Video Tech, Vol 6, no. 3, pp 243-250, June 1996.

[14] 14. Said and A. Pearlman, "An Image Multiresolution Representation for Losssless and Lossy Compression." IEEE Trans. Image Processing, Vol. 5, No. 9, pp 243-250, Sept. 1996.

Dr..A.G. Ananth was born on 3/11/1947, at Bangalore, India. He received M.Sc degree in Nuclear Physics in 1969 from Bangalore University, India.

In 1975, the Physical Research Laboratory Ahmadabad awarded him $\mathrm{PhD}$ degree in Space physics. He served as Deputy Director at Indian space research organization, Bangalore. Presently working as Professor in Telecommunication Engineering Department, R.V College of Engineering, Bangalore. His research interests include Space Applications, Image processing, Image compression, MIMO Systems and Satellite communications.

Nagamani.K was born on 30/05/1973 in Bangalore, India. She received B.E degree in 1996 from Bangalore University and M.Tech degree in 2006 from Visveswaraih Technical University, Belgaum, India.

She is pursuing $\mathrm{PhD}$ Degree. Presently working as an Assistant Professor, in Telecommunication Engineering Department at R.V. College of Engineering, Bangalore. Her research interests include Digital Compression techniques and Embedded System design. 